MATEC Web of Conferences 1, 01006 (2012)

DOI: $10.1051 /$ matecconf/20120101006

(c) Owned by the authors, published by EDP Sciences, 2012

\title{
Multiobjective structural damage identification in uncertain environments
}

\author{
R. Perera ${ }^{1}$, E. Sevillano ${ }^{1}$ and A.Ruiz ${ }^{2}$ \\ ${ }^{1}$ Department of Structural Mechanics, Technical University of Madrid, Spain \\ ${ }^{2}$ Department of Applied Mathematics, Technical University of Madrid, Spain
}

\begin{abstract}
Evolutionary algorithms are suitable to solve damage identification problems in a multiobjective context. However, the performance of these methods can deteriorate quickly with increasing noise intensities originating numerous uncertainties. In this paper, a statistic structural damage detection method formulated in a multiobjective context is proposed. The statistic analysis is implemented to take into account the uncertainties existing in the structural model and measured structural modal parameters. The presented method is verified by a number of simulated damage scenarios. The effects of noise and damage levels on damage detection are investigated.
\end{abstract}

\section{Introduction}

Model updating methods have been often applied to identify damage. In this kind of methods, parameters in a finite element (FE) model related to the structural damage are adjusted so that the model predictions match the measured data in an optimal way [1]. For it, a suitable objective function measuring the agreement between numerical predictions and experimental data should be defined.

However, most real-world problems feature multiple optimization objectives. This is also true in real-world damage identification problems in which the lack of a clear objective function advises simultaneous optimizations of several objectives with the purpose of improving the robustness and performance of the procedure $[2,3]$. A good solution to these multi objective optimization problems should preferably be good regarding all objectives. These objectives cannot be optimized independently, but must be treated as a whole. Evolutionary algorithms are a class of stochastic search methods that have been found to be very efficient and effective in solving complex multiobjective problems where conventional optimization tools fail to work well.

On the other hand, in the structural damage identification problems different degrees of uncertainties can be present which will lead to inaccuracies in the procedure. These uncertainties, known as noise, have their origin in several sources derived from the measurement errors or inaccurate computational models and, even, it can also be intrinsic to the problem. If the noisy optimization problem is treated as if it were deterministic will lead the evolutionary algorithm in a wrong direction and degrade the algorithm's performance being the convergence of the optimisation adversely affected. Therefore, the performance of MOEA deteriorates quickly with increasing noise intensities.

A strategy is proposed in this work to improve the performance and robustness of the damage detection methods in noisy problems. In this strategy, solutions should still work satisfactorily when the design variables change slightly. In the present study only random noises in material properties and measured vibration signals are considered.

\section{Objective functions}

The objective functions selected for the FE model updating method have to reflect the deviation between the numerical prediction and the real behaviour of the structure and, therefore, they should be formulated in terms of the discrepancy between FE and experimental quantities. In this work, due to their high performance, the following functions have been chosen [2]:

$$
\begin{aligned}
& F_{1}=1-\prod_{j=1}^{m} M T M A C_{j}=1-\prod_{j=1}^{m} \frac{\operatorname{MAC}\left(\left\{\phi_{\text {numj }}\right\},\left\{\phi_{\text {exp } j}\right\}\right)}{1+\left|\frac{\lambda_{\text {exp } j}-\lambda_{\text {numj }} \mid}{\lambda_{\text {exp } j}+\lambda_{\text {numj }} \mid}\right|} \\
& F_{2}=1-\prod_{j=1}^{m} \text { MACFLEX } X_{j}=1-\prod_{j=1}^{m} \frac{\left|\left\{F_{\text {num }}\right\}^{T}\left\{F_{\exp j}\right\}\right|^{2}}{\left(\left\{F_{\text {num } j}\right\}^{T}\left\{F_{\text {num } j}\right\}\right)\left(\left\{F_{\exp j}\right\}^{T}\left\{F_{\exp j}\right\}\right)}
\end{aligned}
$$

where $\left\{\phi_{j}\right\}$ is the jth mode shape, $\lambda_{j}=\left(2 \pi f_{j}\right)^{2}$ where $f_{j}$ is the eigenfrequency corresponding to $j$-th mode and 
$\left\{F_{j}\right\}$ is the flexibility vector, collecting the diagonal terms of the flexibility matrix corresponding to the jth mode; MAC is the modal assurance criterion [4] and the subscript num and exp are referred to numerical and experimental values, respectively. Both functions take values between zero and one.

\subsection{Noise}

Ideally, evolutionary algorithms should work on the expected objective functions and not be misled due to the presence of noise. However, the objective functions evaluation is subjected to noise which may come from many different sources such as sensory measurement errors or randomized simulations. Noise can affect clearly to the performance of the identification procedure since in case of noisy objective functions, each evaluation of the same solution results in different objective values. Furthermore, the level of noise intensity will also have a big influence in the predictions. In this study, noise model has been incorporated as an additive perturbation to the original objective functions with the purpose of including in this perturbation all the possible kind of noises. This perturbation has been implemented on the basis of Gaussian noise as an additive normal distributed variable with zero mean and a variance $\sigma^{2}$ representing the level of noise present:

$$
\begin{aligned}
& \bar{F}_{1}=F_{1}+\operatorname{Normal}\left(0, \sigma^{2}\right) \\
& \bar{F}_{2}=F_{2}+\operatorname{Normal}\left(0, \sigma^{2}\right)
\end{aligned}
$$

where $\bar{F}$ denote the objective functions with the noise. The introduction of these uncertainties due to noise entails that, during optimization, the only measurable objective functions values are the stochastic.

\section{Evolutionary multiobjective optimiza- tion}

The presence of multiple objectives in a problem, in principle, gives rise to a set of optimal solutions, known as Pareto-optimal solutions, instead of a single optimal solution. In the absence of any further information, one of these Pareto-optimal solutions cannot be said to be better than the other. This demands a user to find as many Pareto-optimal solutions as possible.

Pareto-based approaches constitute the main motivation for using evolutionary algorithms, such as genetic algorithms, to solve multiobjective optimization problems since in a single run of the algorithm several points of the Pareto-optimal set are found.

Genetic algorithm is a widely applied and efficient method of random search and optimization, the development of which is based on the theory of evolution. Its main features are groups search strategy and information exchange between individuals and moreover, the search does not depend on gradient information. Its basic theory was introduced by Holland [5] and developed in the engineering area by Goldberg's work [6]. Due to its potential for solving multi-objective optimization problems, a wide variety of algorithms has been proposed in the literature [7].

\subsection{Non-dominated Sorting Genetic Algorithm II}

In this paper, the Non-dominated Sorting Genetic Algorithm II (NSGA-II) [8] algorithm has been used. This algorithm, one of the most prominent multiobjective evolutionary algorithms, is a modified version of the NSGA [9] algorithm which can be considered as belonging to the second generation of multi-objective evolutionary algorithms. This revised version is more efficient (computationally speaking) and incorporates a better sorting algorithm that keeps diversity without specifying any additional parameters, and elitism and no sharing parameter needs to be chosen a priori.

The approach is based on several layers of classifications of the individuals. Once the population is initialized and before selection is performed, the population is ranked on the basis of non-domination. The first front being completely non-dominated individuals in the current population and the second front being dominated by the individuals in the first front only and so on. Each individual in each front is assigned rank (fitness) values or is based on the front to which it belongs. Individuals in the first front are given a fitness value of 1 and individuals in the second are assigned a fitness value of 2 and so on.

In addition to a fitness value a new parameter called crowding distance is calculated for each individual. The crowding distance is a measure of how close an individual is to its neighbours. A large average crowding distance will result in better diversity in the population. Parents are selected from the population by using binary tournament selection based on the rank and crowding distance. An individual is selected if the rank is less than the other or if the crowding distance is greater than the other. The selected population generates offsprings from crossover and mutation operators.

The population with the current population and current offsprings is sorted again based on nondomination and only the best $N$ individuals are selected, where $N$ is the population size. The selection is based on rank and the on the crowding distance in the last front. Unlike NSGA, an external memory is not required and elitism is introduced by combining the best parents with the best offspring obtained.

\subsection{Robustness}

In the NSGA II method two additional approaches have been included with the purpose of increasing the performance and robustness of the identification procedure.

On one hand, as commented above, expected fitness functions are not available due to the stochasticity of the problem because of noise. Then, to improve the performance of the method, fitness functions might be estimated by averaging the objective values over a number of randomly sampled disturbances. However, since these disturbances can be chosen deliberately a high 
number of samples in NSGA II might be necessary to get an accurate estimation which results in potentially expensive objective evaluations. Furthermore, by working only with expected objective functions, solutions with high objective variance might be considered as robust since deviations from the true objective functions might cancel one with other in the target point.

In this sense, considering that we are working in a multiobjective framework, the robustness of the method might be improved considerably by including, additionally, objective functions depending on variance as separate optimization criteria. In this way, performance and a robustness measures are optimized simultaneously. The problem to be solved would be a four-objective optimization problem by adding to functions in Eq. (1) and (2) the following two objective functions:

$$
\begin{aligned}
& F_{3}=\frac{\sigma_{F 1}}{\bar{\sigma}} \\
& F_{4}=\frac{\sigma_{F 2}}{\bar{\sigma}}
\end{aligned}
$$

where $\sigma_{F 1}$ and $\sigma_{F 2}$ are the standard deviatiosn obtained from the objective functions values at the sampling points used to get the averaged objective functions and $\bar{\sigma}$ is the average of the standard deviations calculated for each one of the design variables $\mathrm{j}$ at the sampling points.

Extension of NSGA II to consider four objective functions is straightforward.

\section{Numerical simulation study}

In order to investigate the performance of the methodology proposed here, several numerical simulations on a simply supported beam have been made. In all the studies performed, a crossover probability of 0.8 and a mutation probability of 0.01 have been assumed for the GA. In the same way, a binary encoding has been used for the chromosomes of each individual of the population. Each design variable or damage variable $d_{i} \in[0,1] \quad i=1, \ldots, N E$ has been coded into a 3-bit binary number obtaining a resolution of 0.125 , which is acceptable for a suitable estimation of damage. A higher number of bits would result in greater precision but the algorithm convergence (speed) would decrease. Therefore, in order to choose the number of bits per design variable, a compromise should be adopted between precision and convergence.

Using this encoding, damage indices are between 0 and 1 since, once the optimal chromosome has been found, the damage values are obtained through the inverse of the decoded values.

For all cases, the exact solution is compared with the solution by the proposed multi-objective approach. In order to decrease the influence of random effects characterics to the evolutionary algorithm, 5 independent runs were performed per test problem to produce the mean \pm one sample standard deviation plot.

The problem for a comparative investigation consists in identifying damage for a simply supported concrete beam of length $\mathrm{L}=6 \mathrm{~m}$ and rectangular cross section $\mathrm{b} \times \mathrm{h}$ $=0.25 \mathrm{~m} \times 0.2 \mathrm{~m}$. For numerical analysis purposes the beam was divided into 10 two-dimensional beam elements, resulting in a chromosome of 30 bits length. The beam was assumed to have a Young's modulus $\mathrm{E}$ of $30 \mathrm{GPa}$ and a density $\rho$ of $2500 \mathrm{~kg} / \mathrm{m} 3$.

The beam was subjected to a multiple simulated damage scenario (Fig. 1) of complex identification. The "measured" dynamic responses of the beam before and after damage were generated previously. The baseline finite element model of the beam was created using Euler-Bernoulli planar elements with two degrees of freedom per node.

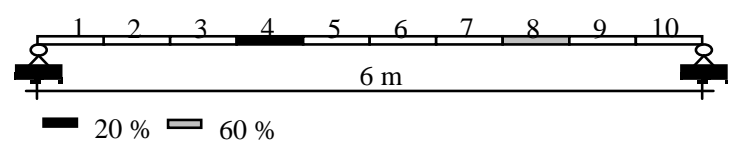

Fig. 1. Multiple damage scenario for the numerical beam

Since the present example is a numerical simulation, the baseline values are perfectly known and, therefore, the possible effect of poorly estimated baseline values is removed from this study. Any parameter estimation differing from the baseline value might be associated with damage.

To be more consistent with the field test conditions and to check the robustness of the proposed procedures, only the four lowest vibration modes were considered, and due to the limited number of sensors, the mode shape vector was only read at a limited number of locations coincident with the vertical degrees of freedom of the nodes in Fig 1. Furthermore, different levels of noise $(1 \%, 5 \%$ and $15 \%)$ were included according to Eq.(3) and (4). The presence of noise can affect the performance of the damage identification procedure especially with increasing noise intensities and, therefore, it is a way of evaluating the performance and robustness of the proposed methodology.

Three different approaches were tested: a) A twoobjective problem solved with NSGA II method; b) A two-objective problem solved with NSGA II method but averaging the objective functions at 5 sample points such as commented in Section 3.2; c) a four-objective problem solved with NSGA II method but averaging the objective functions at 5 sample points such as commented in Section 3.2.

Figs 2, 3 and 4 show the damage distribution for the beam problem when solved with the chosen criteria considering the different levels of noise. The results shown are the average of the 5 optimum solutions for the 5 runs carried out. In the same way, Figs 5 and 6 show the standard deviation for $1 \%$ and $15 \%$ noise. The standard deviation can be useful to measure the sensitivity of each damage parameter to the noise in the "measurements". Sensitive parameters will generally show large standard deviations.

Results demonstrate clearly the improvement obtained when 4 objective functions are used.

Figs 7 and 8 show the probability of damage existence (PDE) for the different elements and for levels of noise $1 \%$ and $15 \%$ by using the procedure shown in [10]. 


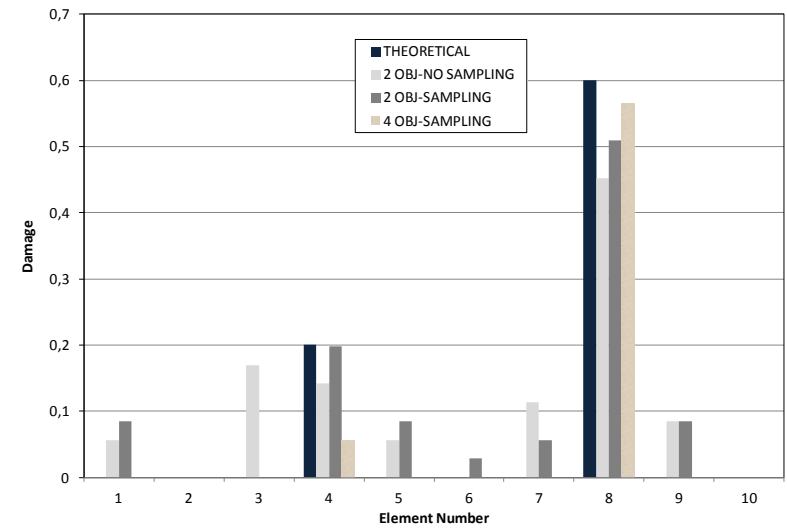

Fig. 2. Damage distribution (1\% noise)

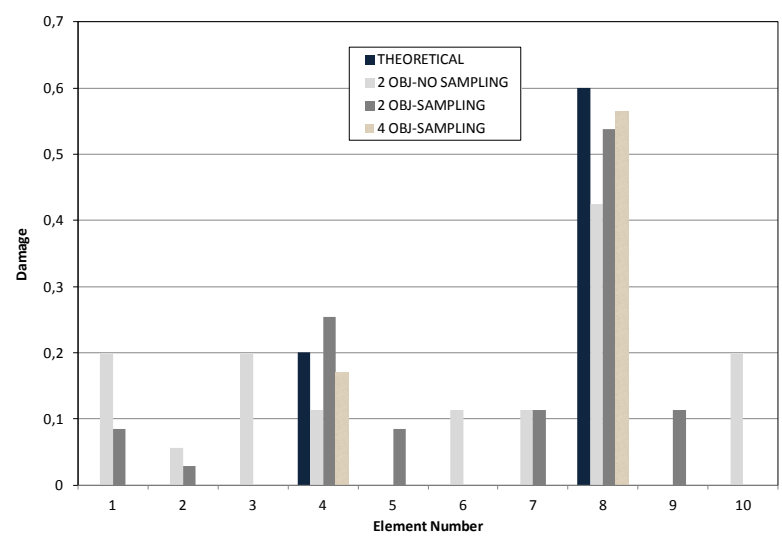

Fig. 3. Damage distribution (5\% noise)

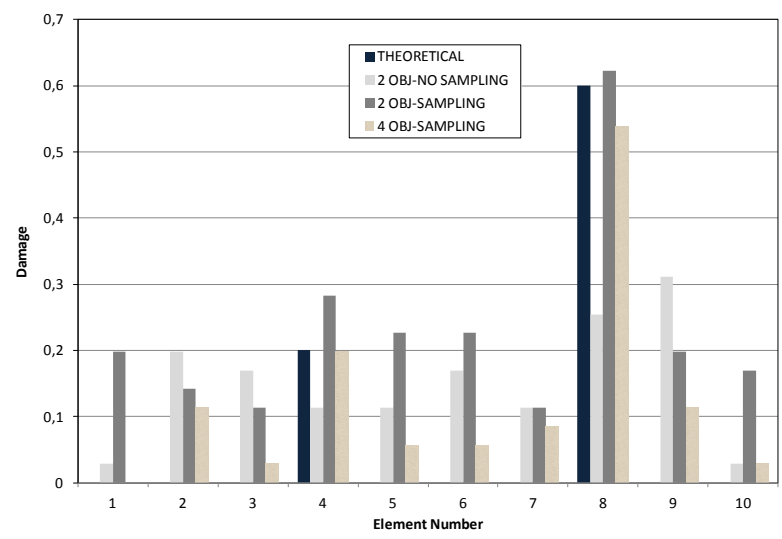

Fig. 4. Damage distribution (15\% noise)

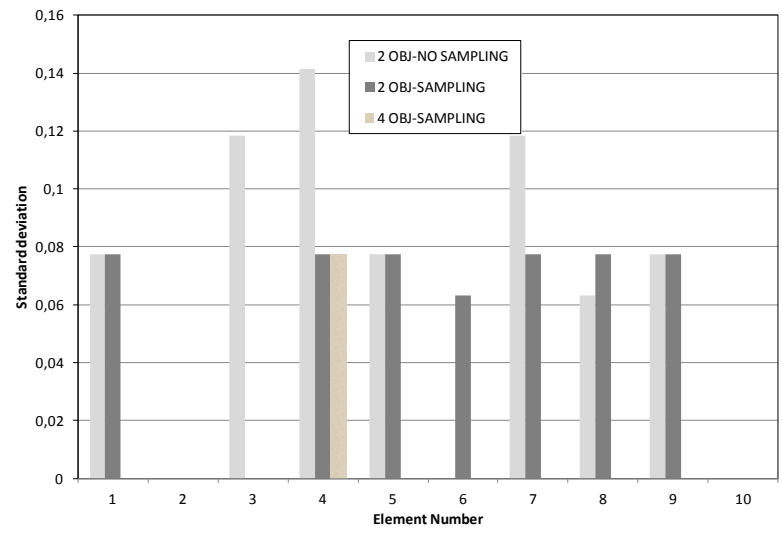

Fig. 5. Standard deviation (1\% noise)

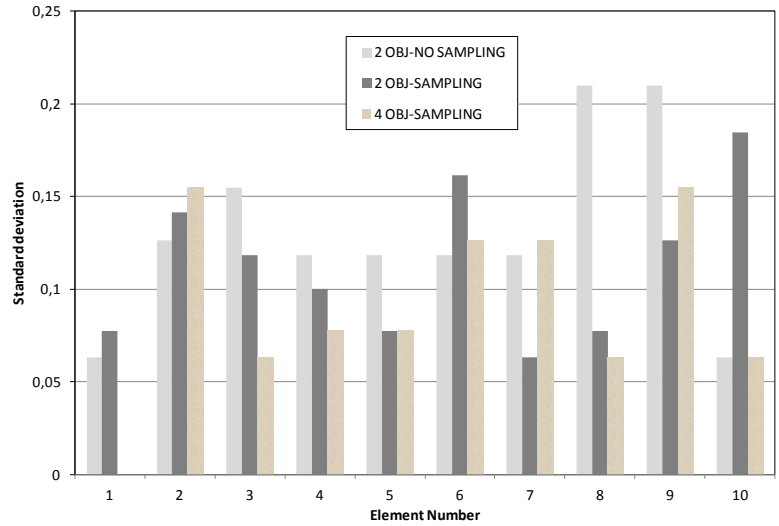

Fig. 6. Standard deviation (15\% noise)

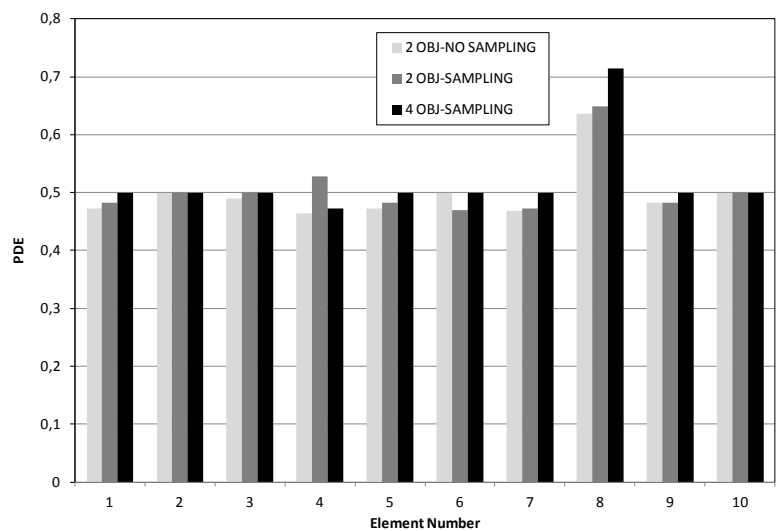

Fig. 7. Probability of damage existence (1\% noise)

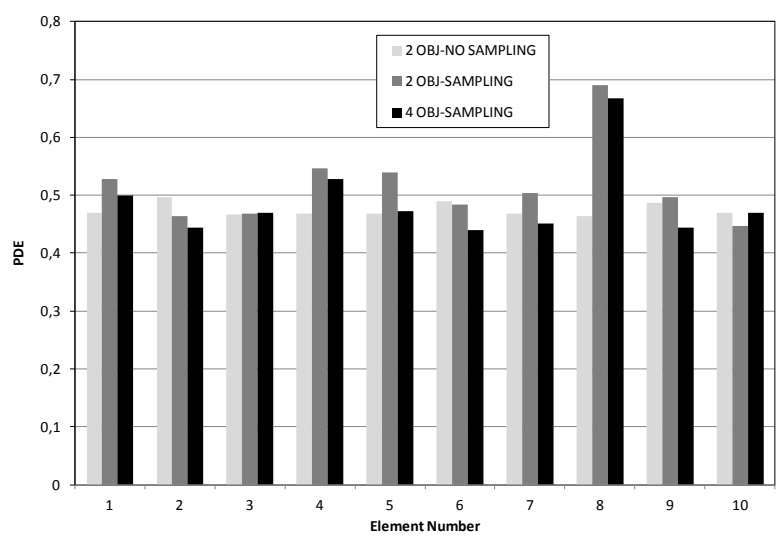

Fig. 8. Probability of damage existence (15\% noise)

\section{Conclusions}

A statistical multiobjective structural damage detection algorithm is developed in this paper. The approach has been focused in the performance and robustness of the damage identification procedure. The uncertainties existing in the structural model and measured structural modal parameters have been taken into account. The probability of damage existence can also be obtained based on the probability density functions of structural stiffness parameters in the intact and damaged state. The presented method has been verified by a numerical study on a simple supported beam with different levels of noise. 


\section{References}

1. M.I. Friswell, Damage identification using inverse methods, Philosophical Transactions of the Royal Society 365, 393 (2007)

2. R. Perera, A. Ruiz, C. Manzano, An evolutionary multiobjective framework for structural damage localization and quantification, Engineering Structures 29, 2540 (2007)

3. S. Jung, S.Y. Ok, J. Song, Robust structural damage identification based on multi-objective optimization, International Journal of Numerical Methods In Engineering 81, 786 (2010)

4. R.J. Allemang, D.L. Brown, A correlation for modal vector analysis, Proceedings of 1 st international modal analysis conference 110 (1982).

5. J. Holland, Adaptation in natural and artificial systems, Cambridge (MA): MIT Press (1975).

6. D. Goldberg, Genetic algorithms in search, optimization and machine learning, Reading (MA): Addison-Wesley (1989).

7. A. Abraham, L. Jain, R. Goldberg, Evolutionary multiobjective optimization: Theoretical advances and applications, Springer-Verlag London (2005).

8. K. Deb, A. Pratap, S. Agarwal, T. Meyarivan, A fast and elitist multi-objective genetic algorithm: NSGAII, IEEE Transactions on Evolutionary Computation 6, 181 (2002).

9. N. Srinivas, K. Deb, Multiobjective Optimization Using Nondominated Sorting in Genetic Algorithms, Evolutionary Computation 2, 221 (1994).

10. Y. Xia, H. Hao, Statistical damage identification of structures with frequency changes, Journal of Sound and Vibration 263, 853 (2003). 